\title{
Quercetin protects RAW264.7 macrophages from glucosamine-induced apoptosis and lipid accumulation via the endoplasmic reticulum stress pathway
}

\author{
XIAXIA CAI $^{1 *}$, LEI BAO $^{2 *}$, XIAOQIAN DAI $^{1}$, YE DING $^{1}$, ZHAOFENG ZHANG $^{1}$ and YONG LI ${ }^{1}$ \\ ${ }^{1}$ Department of Nutrition and Food Hygiene, School of Public Health, Peking University, Beijing 100191; \\ ${ }^{2}$ Department of Clinical Nutrition, Peking University International Hospital, Beijing 102206, P.R. China
}

Received December 4, 2014; Accepted August 24, 2015

DOI: $10.3892 / \mathrm{mmr} .2015 .4340$

\begin{abstract}
It is increasingly recognized that macrophages are a key cell in the development of atherosclerosis. Glucosamine, the product of the hexosamine biosynthetic pathway in diabetes mellitus, can disturb lipid metabolism, induce apoptosis and accelerate atherosclerosis via endoplasmic reticulum (ER) stress in various types of cells. Previous studies have indicated that quercetin possesses antidiabetic, anti-oxidative, anti-inflammatory and anti-apoptotic activities as a flavonoid. Studies have also demonstrated its novel pharmacological properties for inhibiting ER stress. The present study focussed on the effects of quercetin on cell injury and ER stress in glucosamine-induced macrophages. RAW264.7 macrophages were cultured with $15 \mathrm{mM}$ glucosamine, following which the levels of apoptosis, intracellular total and free cholesterol, and apoptosis- and ER stress-associated proteins were measured in the macrophages treated with or without quercetin. Additionally, the ratio of cholestryl ester/total cholesterol was calculated to observe the formation of foam cells. The results demonstrated that apoptosis and abnormal lipid accumulation in the RAW264.7 cells, which was induced by glucosamine,
\end{abstract}

Correspondence to: Professor Yong Li, Department of Nutrition and Food Hygiene, School of Public Health, Peking University, 38 Xueyuan Road, Haidian, Beijing 100191, P.R. China

E-mail: liyongbmu@163.com

*Contributed equally

Abbreviations: AS, atherosclerosis; ATF6, activating transcriptional factor 6; CHOP, C/EBP homologous protein; CVD, cardiovascular disease; DMEM, Dulbecco's modified Eagle's medium; ER, endoplasmic reticulum; GRP78, glucose regulated protein 78; IRE1, inositol requiring enzyme-1; JNK, c-Jun N-terminal kinase; Phospho-JNK, phosphorylated-JNK; TUNEL, terminal-deoxynucleoitidyl transferase mediated dUTP nick end labeling

Key words: quercetin, macrophages, glucosamine, endoplasmic reticulum stress, atherosclerosis were significantly reversed by quercetin. In addition, quercetin treatment suppressed the increase of C/EBP homologous protein, and inhibited the activation of JNK and caspase-12, which was induced by glucosamine. Quercetin also increased the expression level of full length activating transcriptional factor 6 and decreased the expression of glucose regulated protein 78. Of note, the beneficial effects of quercetin on the glucosamine-induced RAW264.7 cells were reversed by treatment with tunicamycin. These findings suggest that quercetin may have properties to prevent glucosamine-induced apoptosis and lipid accumulation via the ER stress pathway in RAW264.7 macrophages.

\section{Introduction}

Diabetes mellitus is the most important risk factor for cardiovascular disease (CVD) (1). The International Diabetes Federation estimates there were $366,000,000$ individuals with diabetes in 2011, and this is expected to rise to $552,000,000$ by 2030 (2). Despite being aware of its severity, the underlying mechanism and therapy of diabetes and its complications remains to be fully elucidated and are underused (2).

Atherosclerosis (AS), the predominant cause of CVD, which causes the majority of diabetes-associated mortality and much of the disability associated with diabetes (3). Macrophages are key in all phases of atherosclerosis, from the formation of the fatty steak to plaque rupture (4). Glucosamine is the product of the hexosamine biosynthetic pathway and, as an effective nutritional supplement used in human osteoarthritis, glucosamine supplementation appears to be safe, with no adverse vascular consequences (4). Orally administered glucosamine sulfate has been observed to exert an anti-atherogenic effect (5). However, in patients with myocardial infarction without diabetes, higher concentrations of glucosamine were noted, compared with those with myocardial infarction and diabetes, suggesting that glucosamine levels may be not directly determined by glucose concentrations (6). There may be other mechanisms (6), and data from animal models and cell culture experiments also support the suggestion that glucosamine disturb lipid metabolism, increase the area of atherosclerotic lesions, induce apoptosis-associated protein expression and accelerate 
atherosclerosis $(7,8)$. These findings suggest that glucosamine is not suitable as an effective preventive strategy for diabetes, however, these changes were independent of blood glucose concentration, glucose tolerance, plasma insulin or plasma lipid levels $(7,8)$. In addition, similar to the above studies, glucosamine has been recognized as a potent inducer of endoplasmic reticulum (ER) stress (9). Therefore, the use of agents against apoptosis, dyslipidemia and ER stress may offer the possibility for improvement of diabetes.

Quercetin, a natural flavonoid, is reported to possess several medicinal and therapeutic properties. Epidemiologic studies have suggested that quercetin may reduce the risk of cardiovascular disease (10-14). It can reduce systolic blood pressure, decrease plasma concentrations of total cholesterol, triglycerides and atherogenic oxidized-low density lipoprotein (ox-LDL), and increase the concentration of high density lipoprotein (HDL)-cholesterol $(10,13,14)$. In addition, the administration of quercetin to mice $(15,16)$ and rabbits $(17,18)$ inhibits the development of atherosclerosis through its anti-oxidant and anti-inflammatory mechanisms. It is also effective in controlling fasting and postprandial blood glucose levels in animal models of diabetes mellitus (19). In vitro studies have demonstrated that activated macrophages may be a potential target of dietary flavonoids in the aorta (20), and they may act work on key atherogenic activities of macrophages, including foam cell formation and pro-oxidant/pro-inflammatory responses (21). Excess or prolonged ER stress leads to cell apoptosis $(22,23)$, and the protective property of quercetin against ER stress induced by ox-LDL, tunicamycin (24) and $\mathrm{Pb}$ (25) have been confirmed. Although quercetin supplementation has been demonstrated to inhibit high glucose-induced apoptosis (26), limited evidence is available regarding the benefits of quercetin on macrophage apoptosis induced by glucosamine, and the mechanisms require further elucidation.

In the present study, $15 \mathrm{mM}$ glucosamine and tunicamycin-treated RAW264.7 macrophages were used to investigate whether quercetin administration results in the alleviation of apoptosis and lipid accumulation, and to examine whether this protective effect of quercetin is, in part, due to the inhibition of ER stress. This aimed to provide the basis for identifying novel molecular targets and therapeutic agents for treatment against diabetes associated atherosclerosis.

\section{Materials and methods}

Materials and reagents. The RAW264.7 mouse macrophage cell line was purchased from the Type Culture Collection of the Chinese Academy of Medical Sciences (Beijing, China). Dulbecco's modified Eagle's medium (DMEM) and fetal bovine serum (FBS) were from Gibco Life Sciences (Carlsbad, CA, USA). Quercetin ( $\geq 95 \%$, high performance liquid chromatography), $N$-Acetyl-D-glucosamine and tunicamycin (from Streptomyces sp.) were obtained from Sigma-Aldrich (St. Louis, MO, USA). An Annexin-V-Fluos staining kit was purchased from Roche Diagnostics (Mannheim, Germany). A One-step Terminal-deoxynucleotidyl Transferase Mediated Nick End Labeling (TUNEL) apoptosis in situ detection kit (cat. no. KGA7061) and poly-L-lycine (cat. no. KGF026) were obtained from KeyGEN Biotech Co., Ltd. (Nanjing, China). The tissue/cell total/free cholesterol assay kits (cat. no. E1015 and E1016) were from Applygen Technologies, Inc. (Beijing, China). Polyclonal antibodies against glucose regulated protein 78 (GRP78; cat. no. 3183), C/EBP homologous protein (CHOP; cat. no. 2895) and $\beta$-actin (cat. no. 4970) were obtained from Cell Signaling Technology, Inc. (Danvers, MA, USA); those against c-Jun N-terminal kinase (JNK) (sc-7345), phosphorylated (p-)JNK (cat. no. sc-6254) and caspase-12 (cat. no. sc-5627) were obtained from Santa Cruz Biotechnology, Inc. (Dallas, TX, US), and antibody against activating transcriptional factor $6 \alpha$ (cat. no. ATF6 $\alpha$; full length) was obtained from Acris Antibodies $\mathrm{GmbH}$ (Herford, Germany). Goat anti-mouse IgG (H+L)-horeseradish peroxidase (HRP; cat. no. ZB-2305) and goat anti-rabbit IgG (H+L)-HRP (cat. no. ZB-2301) were obtained from Beijing Zhongshan Golden Bridge Biotchnology Co., Ltd. (Beijing, China).

Cell culture and treatment. The RAW264.7 cells were cultured in DMEM with $10 \% \mathrm{FBS}$ at $37^{\circ} \mathrm{C}$ in a humidified, $5 \% \mathrm{CO}_{2}$ atmosphere. The cells were subcultured in culture flasks (Corning Incorporated, Corning, NY, USA) and passaged every 3-4 days. The cells were cultured in serum-free medium for $12 \mathrm{~h}$ prior to the subsequent intervention once grown to $70 \%$ confluence. The cells were treated with $15 \mathrm{mM}$ glucosamine, with or without different doses of quercetin $(5,10$ and $20 \mu \mathrm{M}$ ) for $24 \mathrm{~h}$. In another group of experiments, the cells were pretreated with $5 \mu \mathrm{g} / \mathrm{ml}$ tunicamycin for $4 \mathrm{~h}$ followed by treatment with glucosamine and quercetin. Cells incubated in normal medium (DMEM with $10 \%$ FBS) for $24 \mathrm{~h}$ were used as a vehicle group, and cells treated with $15 \mathrm{mM}$ mannitol, instead of glucosamine, for $24 \mathrm{~h}$ were used as an osmolarity control. All the above cells were incubated at $37^{\circ} \mathrm{C}$ in a humidified, $5 \% \mathrm{CO}_{2}$ atmosphere.

Flow cytometric analysis. Following treatment in $60 \mathrm{~mm}$ culture dishes, the cells $\left(1 \times 10^{6}\right)$ were digested using the trypsin enzymwithout EDTA, following which were harvested, washed with phosphate-buffered saline (PBS) and centrifuged at $200 \mathrm{x} \mathrm{g}$ for $5 \mathrm{~min}$. The cell pellet was then resuspended in $100 \mu \mathrm{l}$ of Annexin-V-Fluos labeling solution, and incubated for $15 \mathrm{~min}$ at $25^{\circ} \mathrm{C}$. Finally, the cells were analyzed on a flow cytometer (FACSCalibur, BD Biosciences, Franklin Lakes, NJ, USA) at $488 \mathrm{~nm}$ excitation, with a $515 \mathrm{~nm}$ bandpass filter for fluorescein detection and a filter $>600 \mathrm{~nm}$ for propidium iodide (PI) detection.

TUNEL staining. DNA fragmentation provides markers of apoptosis, which was assessed in the present study using a TUNEL assay. The cells $\left(2.5 \times 10^{6}\right)$ were treated on cover slips with poly-L-lycine in 6 -well plates, and were fixed in $4 \%$ paraformaldehyde for $30 \mathrm{~min}$ at room temperature. The cells were then washed three times with $1 \mathrm{X}$ PBS, and $1 \%$ Triton-X-100 was added for 5 min to promote permeability, followed by washing with $1 \mathrm{X}$ PBS. The cells were then blocked with $3 \% \mathrm{H}_{2} \mathrm{O}_{2}$, and incubated with $50 \mu \mathrm{l}$ TdT enzyme reaction solution, containing $45 \mu \mathrm{l}$ equilibration buffer, $1.0 \mu \mathrm{l}$ TRITC-5-dUTP (cat. no. KGA7061; KeyGEN Biotech Co., Ltd.) and $4.0 \mu \mathrm{l} \mathrm{TdT}$ enzyme, for $60 \mathrm{~min}$ at $37^{\circ} \mathrm{C}$ in the dark. Using $543 \mathrm{~nm}$ excitation and $571 \mathrm{~nm}$ detection wavelengths, the fluorescence intensity was analyzed using a fluorescence 
microscope (Nikon Eclipse TE2000-S; Nikon Corporation, Tokyo, Japan), with apoptotic cells exhibiting intense red fluorescence.

Intracellular total cholesterol and free cholesterol assay. The accumulation of cholesterol in the cells is closely associated with the formation of foam cells. Following starvation for $12 \mathrm{~h}$, the cells were treated with glucosamine and quercetin for $24 \mathrm{~h}$, and then co-incubated with $50 \mathrm{mg} / \mathrm{l} \mathrm{ox}-\mathrm{LDL}$ for $12 \mathrm{~h}$. Following the treatments described above, the cells were washed three times with $1 \mathrm{X}$ PBS, and $1 \times 10^{6}$ cells were lysed in $0.1 \mathrm{ml}$ lysis buffer provided in the tissue/cell total/free cholesterol assay kits (cat. nos. E1015 and E1016; Applygen Technologies, Inc.). The total lysates were centrifuged at 2,000 g for $5 \mathrm{~min}$, and the supernatant was harvested and divided into three equal volumes to be prepared for the measurement of protein concentration, total cholesterol (TC) and free cholesterol (FC) content. The samples $(10 \mu \mathrm{l})$ were then assayed for TC and FC using $190 \mu 1$ enzyme regent of the GPO Trinder enzymatic reaction in a 96-plate well. The absorbtion was measured at $550 \mathrm{~nm}$ on a BioTek EON ${ }^{\mathrm{TM}}$ plate reader (BioTek Instruments, Inc., Winooski, VT, USA). Protein content was determined using a Bicinchoninic Acid (BCA) Protein Assay kit (Beyotime Institute of Biotechnology, Haimen, China), with the concentrations of TC and FC expressed as $\mathrm{nmol} / \mathrm{mg}$ total cellular protein.

Western blot analysis. Following treatment for $24 \mathrm{~h}$ in $10 \mathrm{~mm}$ culture dishes, the cells $\left(1 \times 10^{7}\right)$ were washed three times with ice-cold 1X PBS and lysed for $30 \mathrm{~min}$ in lysis buffer with $1 \mathrm{mM}$ phenylmethylsulfonyl fluoride (cat. no. 78830; Sigma-Aldrich). The total protein lysates were centrifuged at $12,000 \mathrm{~g}$ for $5 \mathrm{~min}$ at $4^{\circ} \mathrm{C}$, and the protein content in the supernatants were quantified using a BCA Protein Assay kit. The protein samples $(20 \mu \mathrm{g})$ from each group were mixed with $5 \mathrm{X}$ sample loading buffer and were used for western blotting analysis through 8,10 or $12 \%$ SDS-PAGE (for GRP78 and ATP $6 \alpha$; JNK, p-JNK, caspase-12 and $\beta$-actin; and CHOP, respectively; cat. no. KGP113K, KeyGEN Biotech Co., Ltd.), followed by transfer onto $0.45-\mu \mathrm{m}$ (pore size) polyvinylidene fluoride membranes (EMD Millipore, Bedford, MA, USA) through a wet transfer method in an ice-bath for $2 \mathrm{~h}$. The membranes were blocked with 5\% (v/v) skim milk at room temperature for $1 \mathrm{~h}$, and incubated with primary antibody diluted with 5\% bovine serum albumin (cat. no. A4737; Sigma-Aldrich) at $4^{\circ} \mathrm{C}$ overnight. The following primary antibodies were used: GRP78, CHOP and ATF6 $\alpha(1: 1,000)$, $\beta$-actin $(1: 2,000)$ and, JNK, p-JNK and caspase-12 (1:200). Following washing the membranes three times, they were incubated with HRP-conjugated secondary antibodies $(1: 4,000)$, diluted with $5 \%$ bovine serum albumin, for $1 \mathrm{~h}$ at $37^{\circ} \mathrm{C}$ and detected using an enhanced chemiluminescence system (ECL; M\&C Gene Technology, Ltd, Beijing, China). Finally, images were captured using a digital camera and were analyzed using ImageJ software (version 1.46r; National Institutes of Health, Bethesda, MD, USA).

Statistical analysis. Data are presented as the mean \pm standard deviation of at least three independent experiments. One-way analysis of variance was used to compare differences, followed by Dunnett-t multiple comparison tests with
SPSS 13.0 for Windows (SPSS, Inc., Chicago, IL, USA). $\mathrm{P}<0.05$ was considered to indicate a statistically significant difference.

\section{Results}

Quercetin protects RAW264.7 cells from apoptosis and cell death induced by glucosamine. The anti-apoptotic effect of quercetin against glucosamine in RAW264.7 cells was evaluated using Annexin-V-FITC/PI double staining and detection using flow cytometry. As shown in Fig. 1A and B, the treatment of RAW264.7 cells with $15 \mathrm{mM}$ glucosamine for $24 \mathrm{~h}$ induced apoptosis, compared with the vehicle control cells $(6.16 \pm 0.04$, vs. $0.13 \pm 0.02 \%$, respectively), however, quercetin $(5,10$ and $20 \mu \mathrm{M}$ ) significantly attenuated glucosamine-induced apoptosis in a dose dependent manner. Consistent with the above, in the RAW264.7 cells stained with TUNEL, the number of positive cells was markedly higher in the glucosamine-induced cells, compared with the vehicle control cells. Representative photomicrograph are presented in Fig. 1C, with the red TUNEL-positive cells clearly marked.

In addition to its anti-apoptotic property, the present study also determined the protective effect of quercetin on glucosamine-induced cell death (Fig. 1A and B). The addition of glucosamine at $15 \mathrm{mM}$ for $24 \mathrm{~h}$ increased cell death, compared with the vehicle group $(16.87 \pm 0.21$, vs. $2.55 \pm 0.16 \%$, respectively). However, incubation of the glucosamine-induced cells with different doses of quercetin $(5,10$ and $20 \mu \mathrm{M})$ resulted in a dose-dependent decrease in the number of necrotic cells.

Quercetin attenuates glucosamine-induced lipid accumulation in RAW264.7 cells. Foam cell formation is a key characteristic of AS (27) and can be induced by excess ox-LDL. To determine the effect of quercetin on glucosamine-induced foam cell formation in the present study, the RAW264.7 macrophages were incubated with $50 \mathrm{mg} / \mathrm{l}$ ox-LDL for $12 \mathrm{~h}$ following glucosamine and quercetin treatment, and subsequent intracellular TC, FC and cholesteryl ester (CE) quantification were performed. Compared with the vehicle control, the addition of ox-LDL to the culture medium including glucosamine amplified foam cell formation as the intracellular TC content (1.96-fold) and FC content (1.74-fold) were significantly increased, the ratio of $\mathrm{CE} / \mathrm{TC}$ was also significantly increased in the glucosamine-treated group. Treatment with quercetin significantly decreased the TC content, FC content and ratio of CE/TC (Fig. 2A-C). However, following treatment with tunicamycin, an ER stress-inducing agent, these effects of quercetin disappeared. Tunicamycin treatment of the cells treated with quercetin and glucosamine led to a significant increase in intracellular TC content and $\mathrm{CE} / \mathrm{TC}$ ratio, compared with those without tunicamycin (Fig. 2A-C). These results indicated that quercetin prevented the amplification of ox-LDL-induced foam cell formation by glucosamine via ER stress in the RAW264.7 cells.

Effect of quercetin on the expression of p-JNK and JNK. JNK activation is known to affect the cell-death mechanism which is involved in ER stress (28-30). To investigate whether JNK was involved in the effect of quercetin on ER stress, the present study detected the expression of pJNK and JNK. The results 
A
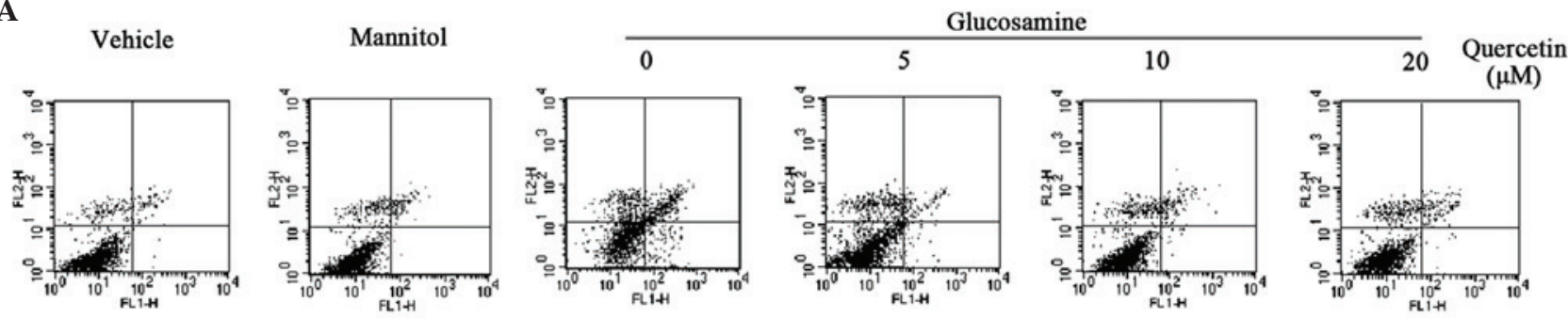

B

\begin{tabular}{|c|c|c|c|c|c|c|}
\hline \multirow{3}{*}{ cells type } & \multirow{3}{*}{ Vehicle } & \multirow{3}{*}{ Mannitol } & \multicolumn{4}{|c|}{ Glucosamine } \\
\hline & & & \multicolumn{4}{|c|}{ Quercetin $(\mu \mathrm{M})$} \\
\hline & & & 0 & 5 & 10 & 20 \\
\hline apoptotic cells (\%) & $0.13 \pm 0.02$ & $0.34 \pm 0.02$ & $6.16 \pm 0.04^{\mathrm{a}}$ & $1.34 \pm 0.07^{b}$ & $0.56 \pm 0.08^{b}$ & $0.55 \pm 0.08^{b}$ \\
\hline necrotic cells (\%) & $2.55 \pm 0.16$ & $4.74 \pm 0.06$ & $16.87 \pm 0.21^{\mathrm{a}}$ & $3.65 \pm 0.08^{b}$ & $4.95 \pm 0.16^{b}$ & $4.72 \pm 0.09^{b}$ \\
\hline
\end{tabular}

C
Vehicle
Mannitol

$$
0
$$

Glucosamine
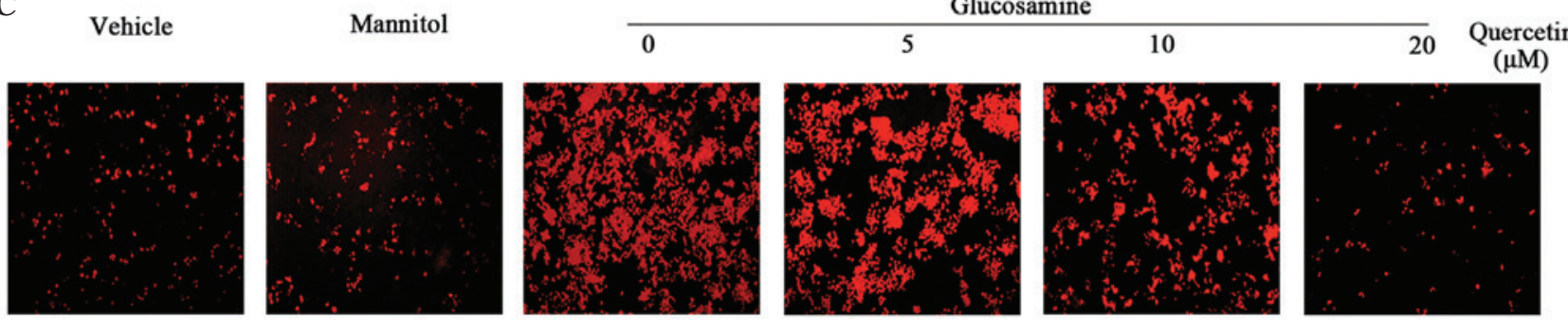

Figure 1. Effects of quercetin on glucosamine-induced apoptosis in RAW264.7 macrophages. (A) Apoptotic cells were stained with Annexin-V-Fluos and PI, and detected using flow cytometry. Flow cytometric analysis clearly differentiated living cells with low Annexin and low PI staining, apoptotic cells with high Annexin and low PI staining, and necrotic cells with high Annexin and high PI staining. (B) Representative data of flow cytometric analysis. The average rate of apoptotic and necrotic cells are presented as the mean \pm standard deviation of at least three independent experiments. (C) Apoptotic RAW264.7 cells were observed using TUNEL staining. Representative images with red TUNEL-positive cells are apoptotic RAW264.7 cells (magnification, $\mathrm{x} 400$ ). ${ }^{\mathrm{a} P}<0.01$, vs. vehicle control; ${ }^{\mathrm{b}} \mathrm{P}<0.01$ vs. glucosamine treatment. PI, propidium iodide; FL1-H, increasing Annexin-V-fluorescence; FL2-H, increasing PI; TUNEL, terminal-deoxynucleotidyl transferase mediated nick end labeling.

demonstrated that $\mathrm{p}-\mathrm{JNK}$ in the glucosamine-treated cells, the active form of JNK, was significantly increased, compared with that of the vehicle control cells $(\mathrm{P}<0.05)$. However, quercetin significantly inhibited the phosphorylation of JNK in a dose-dependent manner. In addition, JNK phosphorylation was not reversed by quercetin in the presence of tunicamycin (Fig. 3A).

Effect of quercetin on the expression levels of $\mathrm{CHOP}$ and caspase-12. In addition to JNK activation, C/EBP homologous proteins and caspase- 12 are the other two apoptotic pathways induced by ER stress (31). To determine the effects of quercetin on CHOP and caspase-12, western blot analysis was performed. Glucosamine treatment resulted in a significant increase in the expression level of CHOP, compared with the vehicle control (23.9-fold). The levels of cleaved caspase-12 were also markedly elevated in the glucosamine-treated cells, compared with the vehicle control $(\mathrm{P}<0.05)$. Notably, 10 and $20 \mu \mathrm{M}$ quercetin significantly decreased the expression of $\mathrm{CHOP}$ and the activation of caspase-12, compared with the glucosamine-treated cells $(\mathrm{P}<0.05)$. No significant changes in the expression levels of CHOP or cleaved caspase-12 were observed between the glucosamine-treated cells and quercetin-treated cells in the presence of tunicamycin ( $\mathrm{P}>0.05$; Fig. 3B).

Quercetin protects against the glucosamine-induced activation of ATF6 $\alpha$ and GRP78. In order to identify the potential effect of quercetin on glucosamine-induced ER stress in RAW264.7 macrophages, the present study measured the expression levels of the ER stress marker, GRP78, and the ER membrane-anchored transcription factor, p90-ATF6 $\alpha$. As shown in Fig. 3C, the glucosamine-treated cells exhibited activation of ER stress markers, with the cells in glucosamine group exhibiting a significant 7.94-fold increase in GRP78, compared with the vehicle control cells. In addition, the expression levels of p90-ATF6 $\alpha$, the inactive form of ATF6 $\alpha$, were markedly decreased in the glucosamine-treated cells, by 0.183 -fold, compared with the vehicle control. Notably, quercetin restored these changes in the levels of GRP78 and p90-ATF6 $\alpha$, whereas tunicamycin suppressed the effects of quercetin on the expression levels of GRP78 and p90-ATF6 $\alpha$ (Fig. 3C).

\section{Discussion}

Animal studies have suggested that hyperglycemia is an independent risk factor of AS. The conditions of hyperglycemia give rise to the accumulation of glucosamine in the vessel wall and accelerate AS $(8,32)$. In human aortic smooth muscle cells (HASMC), monocytes and hepatocytes, glucosamine disturbs lipid metabolism, leading to the accumulation of cholesterol in cultured cells (8), and lipid deposition and macrophage apoptosis are key in the development of AS (33-35). Thus, the inhibition of lipid dysregulation and macrophage apoptosis 
A

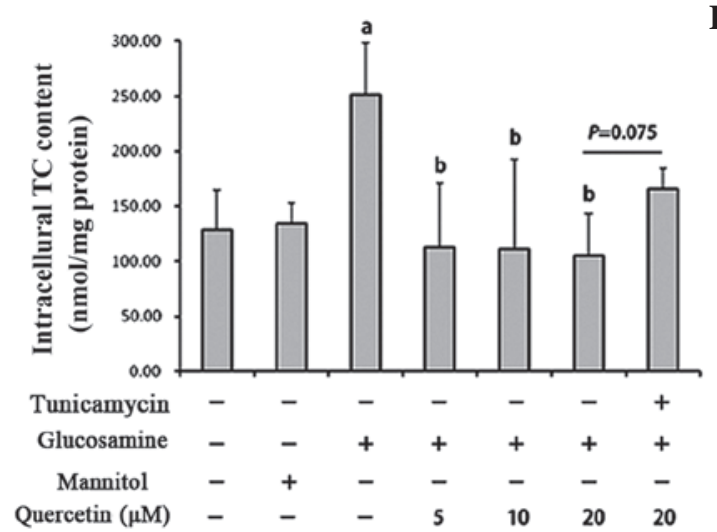

B

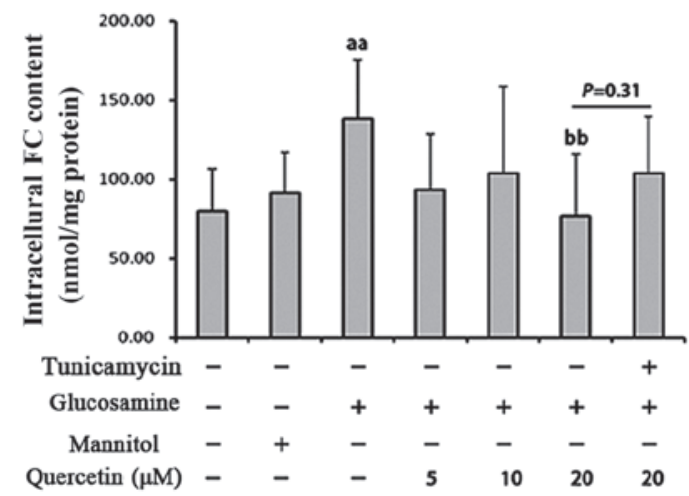

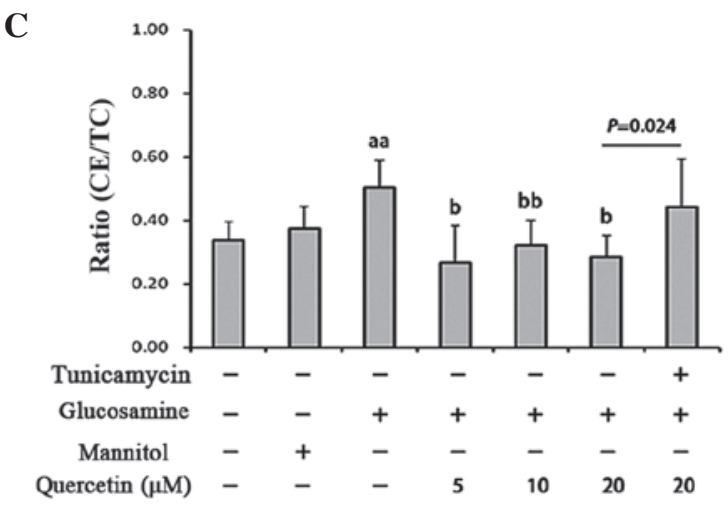

Figure 2. Effect of quercetin on glucosamine-induced intracellular lipid accumulation in RAW264.7 macrophages. Cells were treated with glucosamine $(15 \mathrm{mM})$ in the absence or presence of different concentrations of quercetin $(5,10,20 \mu \mathrm{M})$ for $24 \mathrm{~h}$. In addition, another group of cells were treated with glucosamine $(15 \mathrm{mM})$ and quercetin $(20 \mu \mathrm{M})$ for $24 \mathrm{~h}$ with tunicamycin $(5 \mu \mathrm{g} / \mathrm{ml})$ pretreatment for $4 \mathrm{~h}$. All the cells were treated with oxidized-low density lipoprotein (50 mg/1). (A) Intracellular TC content was measured using a tissue/cell total cholesterol assay kit. (B) Intracellular FC content was measured using a tissue/cell free cholesterol assay kit. (C) CE/TC, calculated as (TC content - FC content) / TC content. Data are presented as the mean \pm standard deviations of six independent experiments and normalized with the protein quantity of each group. ${ }^{\text {a }} \mathrm{P}<0.01$ and ${ }^{\text {aa }} \mathrm{P}<0.05$, vs. vehicle control; ${ }^{\text {b }} \mathrm{P}<0.01$ and ${ }^{\text {bb }} \mathrm{P}<0.05$, vs. glucosamine treatment. TC, total cholesterol; FC, free cholesterol; CE, cholesteryl ester.

may be effective strategy in preventing the development and progression of diabetic atherosclerosis. Quercetin is reported to possess properties against diabetes and CVD $(10-14,19)$. It can also suppress macrophage apoptosis and foam cell formation induced by tunicamycin and ox-LDL (24). However, the effect of quercetin on glucosamine-induced lipid dysregulation and macrophage apoptosis, and the possible mechanism remained to be elucidated. The present study demonstrate that quercetin attenuated glucosamine-induced apoptosis in RAW264.7 macrophages, and suppressing the ER stress pathway by inhibiting ATF6 activation may be one of the mechanisms. In addition, quercetin prevented the formation of foam cells through the ER stress pathway, which may be another cytoprotective mechanism.

Lipid accumulation is one of the predominant characteristics of AS. Free cholesterol loading has been demonstrated to be associated with the activation and apoptosis of macrophages (33). Although a previous study demonstrated that intraperitoneal glucosamine decreased AS in $\mathrm{ApoE}^{-/}$mice (36), glucosamine supplementation has been observed to increase the initiation of AS after 5 weeks, and cholesterol and triglyceride concentrations increase significantly following glucosamine treatment for 20 weeks (4). HASMC and HepG2 cells treated with glucosamine exhibit increased intracellular levels of free cholesterol (8). Quercetin has been reported to interfere with key atherogenic progresses of macrophages, including foam cell formation (21), anti-oxidation (37), anti-inflammation (17) and the regulation of several genes involved in lipid metabolism $(20,38)$. The present study demonstrated that the effects of $50 \mathrm{mg} / \mathrm{l}$ ox-LDL added to macrophages were aggravated by glucosamine treatment, as intracellular TC and FC contents increased markedly following glucosamine-treatment, with a concomitant increase in the CE rate of TC (50.5\%). These results were characteristic of foam cells (39). By contrast, the supplementation of quercetin to the glucosamine and ox-LDL-induced macrophages alleviated ox-LDL uptake and suppressed the formation of foam cells in the RAW264.7 cells. Of note, classical ER-stress inducer treatment reversed the beneficial effects of quercetin on ox-LDL uptake and the formation of foam cells. These results suggested that ER stress may be involved in the glucosamine-induced dysregulation of lipid metabolism.

Increasing evidence supports the suggestion that glucosamine induces the apoprosis of various cels, including rat mesangial cells (40) and pancreatic $\beta$ cells (41). Although limited information is available describing the harmful effects of glucosamine on macrophages, elevated glucose increases flux through the hexosamine biosynthetic pathway, which ultimately leads to increased glucosamine concentrations (42-44). In a study investigating subjects with type 2 diabetes, increased levels of macrophage apoptosis were observed in their atherosclerotic lesions, and this may be a risk factor of plaque destabilization 
A
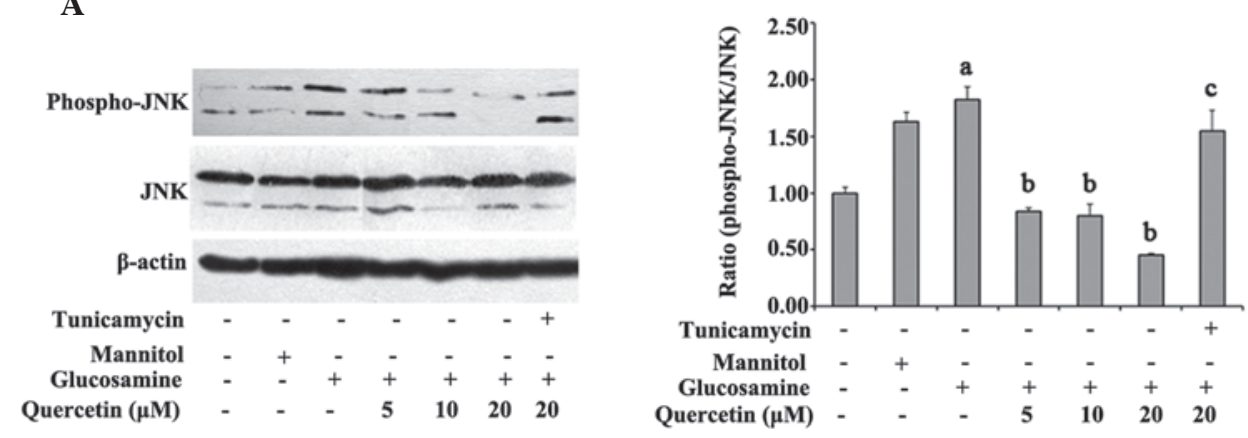

$\mathbf{B}$
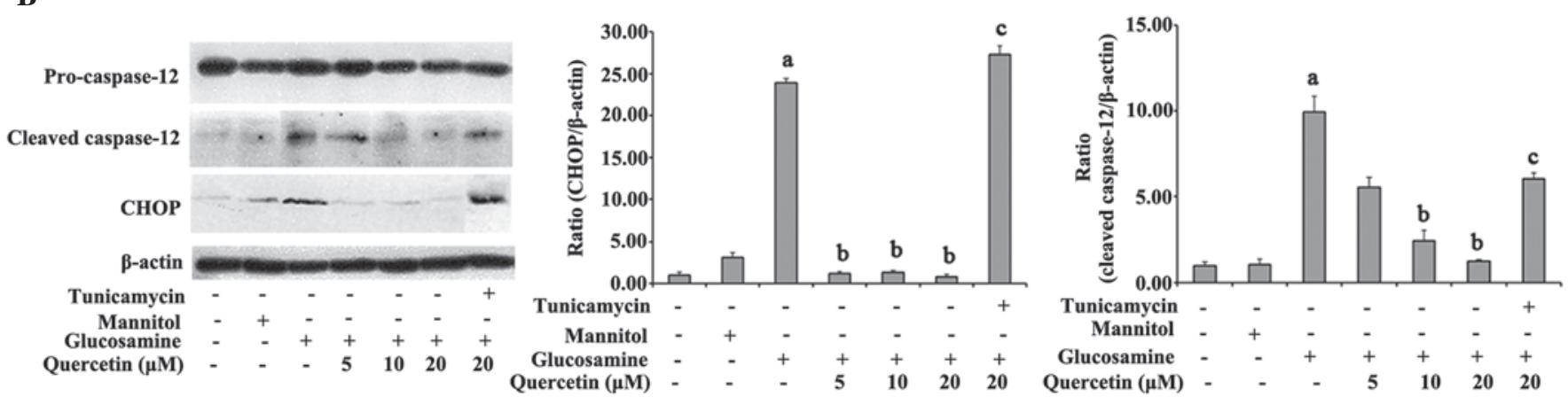

C
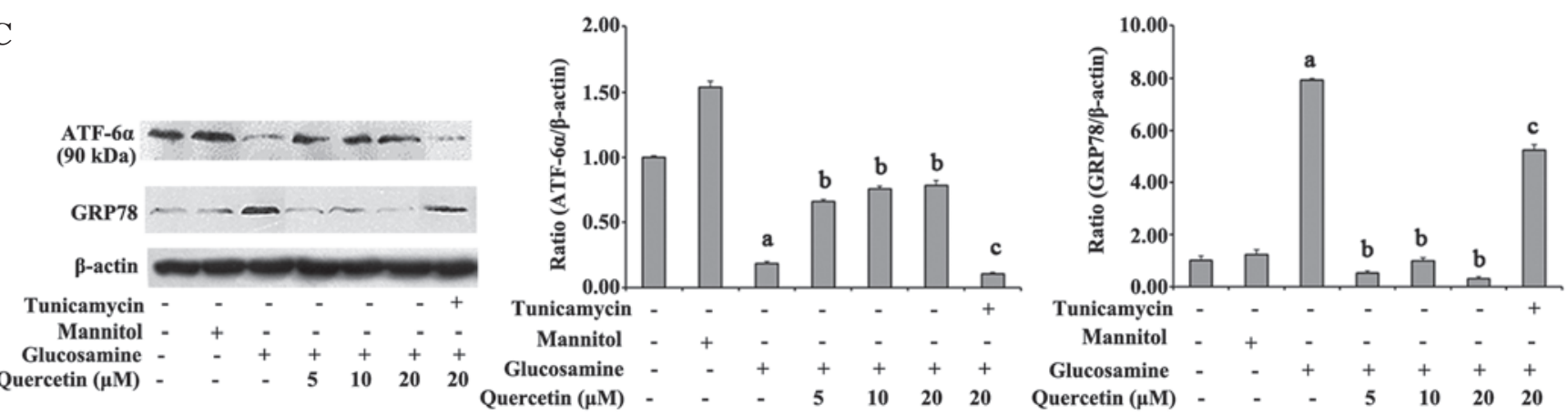

Figure 3. Effects of quercetin on apoptosis- and ER stress-associated proteins in glucosamine-induced RAW264.7 macrophages. (A) Expression levels of phospho-JNK and JNK were measured using western blotting. (B) Expression levels of CHOP and cleaved-caspase-12 were measured using western blotting and normalized to the levels of $\beta$-actin. (C) Expression levels of GRP78 and ATF6 $\alpha$ (90 KDa) were measured using western blotting and normalized to the levels of $\beta$-actin. Data are presented as the mean \pm standard deviations of at least three independent experiments. ${ }^{\text {a }} \mathrm{P}<0.01$, vs. vehicle-treated control; ${ }^{b} \mathrm{P}<0.01$, vs. glucosamine treatment; ${ }^{\circ} \mathrm{P}<0.01$, vs. quercetin $(20 \mu \mathrm{M})$ treatment. ER, endoplasmic reticulum; JNK, c-Jun N-terminal kinase; phospho-JNK, phosphorylated-JNK; CHOP, C/EBP homologous protein; GRP78, glucose regulated protein 78; ATF6, activating transcriptional factor 6.

and acute clinical events (45). In addition, Khan (32) detected increased O-GlcNAc staining, an indicator of intracellular glucosamine concentration (46), in macrophage/foam cells of the atherosclerotic lesions in hyperglycemic ApoE-/- mice, compared with normoglycemic rice. As mentioned previously, quercetin has been observed to prevent macrophages from apoptosis induction by high glucose (26), ox-LDL (24) and $\mathrm{H}_{2} \mathrm{O}_{2}$ (37). However, whether quercetin inhibits glucosamine-induced macrophage apoptosis had not previously reported. The results of the present study suggested that macrophages treated with $15 \mathrm{mM}$ glucosamine for $24 \mathrm{~h}$ exhibited increased apoptotic and necrotic rates, compared with cells in the vehicle control group. However, the addition of quercetin to the glucosamine-treated macrophages significantly decreased the apoptotic and necrotic rates of the cells.
It has been reported that quercetin protects multiple types of cells from apoptosis through various mechanisms. Roshanzamir et al (47) reported that quercetin inhibits $\mathrm{H}_{2} \mathrm{O}_{2}$-induced SK-N-MC cell death through the downregulation of HIF-1 $\alpha$, Foxo-3a and NICD, as well as pro-apoptotic factors, including p53 and Bax. Suganya et al (48) observed that quercetin has an anti-apoptotic effect against tunicamycin-induced endothelial cell toxicity through the ER-stress/CHOP pathway, which involved an increase in the expression of B cell lymphoma-2 (Bcl-2) and reduction in the expression of Bcl-2-associated X protein (Bax). Similar results have been observed in RAW264.7 macrophages (24). Our previous study suggested that quercetin prevents $\beta$-cell death through the mitochondrial pathway and nuclear factor- $\kappa \mathrm{B}$ signaling in RINm5F rat insulinoma cells (49). Therefore, 
the biological effects of quercetin on cells remain to be fully elucidated. In the present study, it was observed that quercetin significantly reduced the expression of CHOP and suppressed the phosphorylation of JNK and activation of caspase-12 by inhibiting the expression of GRP78 and activation of ATF6 $\alpha$ in the glucosamine-treated RAW264.7 macrophages. Tunicamycin is a typical ER stress inducer via interfering with N-linked protein glycosylation in ER (50). The present study also found that quercetin did not attenuate glucosamine-induced lipid accumulation in tunicamycin-pretreated cells. Compared with the glucosamine-induced cells without tunicamycin, quercetin had no significant effect on the expression of CHOP, the activities of JNK and caspase-12, GRP78 or full length ATF6 $\alpha$ following tunicamycin treatment. However, no detailed indicator of ER stress-lipid metabolism or other ER stress markers, including as protein kinase-like ER kinase or intercellular adhesion molecule-1 (IRE1) were detected. These data indicated that the ER stress pathway is involved in the beneficial effects of quercetin on glucosamine-induced cell damage.

The ER is located in the cytoplasm close to the cell nucleus, and is a vital organelle responsible for protein biosynthesis, post-translational modifications, folding and assembly of polypeptide chains, intracellular calcium storage and controlling lipid biosynthesis (51). Multiple stimuli, including hyperglycemia (52), hypoxia (53) and chemical toxicity (54) can lead to ER dysfunction and ER stress, which is an adaptive signaling mechanism, termed the unfolded protein response, to restore ER homeostasis. However, continuous and serious ER stress triggers a series of processes necessary for apoptosis, including CHOP, JNK and caspase-12 signaling $(55,56)$. Accumulated evidence indicates the role of $\mathrm{CHOP}$ on various aspects of cell damage associated with ER stress $(57,58) . \mathrm{CHOP}^{-/}$animals exhibit four-fold lower levels of TUNEL-positive cells in the proximal tube epithelium (57). $\mathrm{CHOP}^{-/ /} / \mathrm{ApoE}^{-/-}$mice exhibit minimal apoptosis of macrophages in plaques and, compared with high cholesterol-fed $\mathrm{CHOP}^{-/ /} / \mathrm{ApoE}^{-/-}$mice, high cholesterol-fed $\mathrm{CHOP}^{+/+} / \mathrm{ApoE}^{-/-}$mice exhibit increased rupture of atherosclerotic plaques. Tsukano et al (58) suggested that ER stress-CHOP-Bax-mediated apoptosis contributes to these changes. JNK activation is key in the process of cell apoptosis induced by ER stress (28). In response to ER stress, IRE-1 $\alpha$ activates JNK directly (59). Caspase-12, a caspase localized to the ER, is activated to generate cleaved caspase-12 induced by ER stress (60). Nakagawa et al (61) suggested that treatment of PC12 cells or embryonic fibroblast cells with tunicamycin and thapsigargin induces effective cleavage of caspase-12. Furthermore, in vivo, caspase-12/- mice were found to be resistant to ER stress-induced apoptosis. In the present study, it was demonstrated that glucosamine-treatment increased the expression of CHOP, induced the cleavage of caspase-12 and activated JNK in the RAW264.7 cells. However, quercetin significantly inhibited the cleavage of caspase-12 and the activation of JNK, and suppressed the expression levels of CHOP, whereas these changes were reversed by tunicamycin, which has been used in the analyses of CHOP- or caspase-12-deficient mice $(57,61)$. Therefore, the effects of quercetin contributed to the recovery of RAW264.7 cell damage by glucosamine.
ATF6 is one of three ER stress sensors, and is an ER-resident transmembrane protein, which is vital for proper protein folding and assembly $(55,62)$. ER stress results in the dissociation of GRP78 from the ER membrane proteins, including ATF6, and activated ATF6 translocates to the golgi for cleavage and causes activation of downstream signaling pathways (51). ATF6 consists of ATF6 $\alpha$ and ATF6 $\beta$ in mammalian cells, however, previous studies have suggested that ATF6 $\alpha$ is more sensitive to ER stress $(63,64)$, Rutkowski et al (65) observed that ATF6 $\alpha$-deficient mice were induced with unresolved ER stress following tunicamycin injection, and these alterations increased the expression of CHOP. Yamamoto et al (64) demosntrated that the levels of triacylglycerol and TC in the liver of ATF $6 \alpha^{-/-}$mice were significantly increased, compared with those of ATF $6 \alpha^{+/+}$ mice following tunicamycin injection, and that tunicamycin injection caused the accumulation of neutral lipids in the liver of the ATF $6 \alpha^{-/}$mice. Yao et al (24) suggested that quercetin inhibits the translocation of ATF6 from the cytoplasm into the nucleus in RAW264.7 cells. In the present study, it was revealed that tunicamycin treatment also caused significant lipid accumulation in glucosamine-treated cells treated with quercetin. Notably, glucosamine treatment significantly increased the expression of GRP78 and induced the activation of ATF6 $\alpha$, and decreased the expression levels of $90 \mathrm{kDa}$ ATF6 $\alpha$ (full-length), which is an inactive form of ATF6 $\alpha$. However, the expression of ATF6 $\alpha$ in the quercetin-treated RAW264.7 cells were increased significantly, compared with the glucosamine-treated cells. Quercetin also alleviated the expression of GRP78, whereas, tunicamycin treatment inhibited these effects of quercetin.

In conclusion, the present study suggested that apoptosis and lipid accumulation in RAW264.7 cells induced by glucosamine were partially due to ER stress. Quercetin supplementation inhibited ER stress by decreasing the expression of CHOP, and inhibiting the activation of JNK and caspase-12. Although there may be other mechanisms, the results suggested that the inhibitory effects of quercetin on GRP78 and ATF6 $\alpha$ activation contributed, at least in part, to the above alterations. These findings support the anti-atherosclerotic effects and mechanisms of quercetin upon diabetes in vitro. However, a series of questions remain due to the complex and unclear mechanism of ER stress. Further in vitro and vivo investigations on the effect of quercetin on ER stress are necessary to further elucidate the mechanisms of atherosclerosis in diabetes and the identification of novel targets for therapy.

\section{Acknowledgements}

This study was supported by a research grant from the National Natural Science Foundation of China (grant. no. 81172652).

\section{References}

1. Stamler J, Vaccaro O, Neaton JD and Wentworth D: Diabetes, other risk factors and 12-yr cardiovascular mortality for men screened in the multiple risk factor intervention trial. Diabetes Care 16: 434-444, 1993.

2. Whiting DR, Guariguata L, Weil C and Shaw J: IDF diabetes atlas: Global estimates of the prevalence of diabetes for 2011 and 2030. Diabetes Res Clin Pract 94: 311-321, 2011. 
3. Beckman JA, Creager MA and Libby P: Diabetes and atherosclerosis: Epidemiology, pathophysiology and management. JAMA 287: 2570-2581, 2002.

4. Tannock LR, Kirk EA, King VL, LeBoeuf R, Wight TN and Chait A: Glucosamine supplementation accelerates early but not late atherosclerosis in LDL receptor-deficient mice. J Nutr 136: 2856-2861, 2006.

5. Largo R, Martínez-Calatrava MJ, Sánchez-Pernaute O, Marcos ME, Moreno-Rubio J, Aparicio C, Egido J and Herrero-Beaumont G: Effect of a high dose of glucosamine on systemic and tissue inflammation in an experimental model of atherosclerosis aggravated by chronic arthritis. Am J Physiol Heart Circ Physiol 297: H268-H276, 2009.

6. Nowak A, Szcześniak L, Rychlewski T, Dylewicz P and Przywarska I: Glucosamine levels in people with ischaemic heart disease with and without type II diabetes. Pol Arch Med Wewn 100: 419-425, 1998.

7. Beriault DR, Sharma S, Shi Y, Khan MI and Werstuck GH: Glucosamine-supplementation promotes endoplasmic reticulum stress, hepatic steatosis and accelerated atherogenesis in apoE-/mice. Atherosclerosis 219: 134-140, 2011.

8. Werstuck GH, Khan MI, Femia G, Kim AJ, Tedesco V, Trigatti B and Shi Y: Glucosamine-induced endoplasmic reticulum dysfunction is associated with accelerated atherosclerosis in a hyperglycemic mouse model. Diabetes 55: 93-101, 2006.

9. Qiu W, Avramoglu RK, Rutledge AC, Tsai J and Adeli K: Mechanisms of glucosamine-induced suppression of the hepatic assembly and secretion of apolipoprotein B-100-containing lipoproteins. J Lipid Res 47: 1749-1761, 2006.

10. Egert S, Bosy-Westphal A, Seiberl J, Kürbitz C, Settler U, Plachta-Danielzik S, Wagner AE, Frank J, Schrezenmeir J, Rimbach G, et al: Quercetin reduces systolic blood pressure and plasma oxidised low-density lipoprotein concentrations in overweight subjects with a high-cardiovascular disease risk phenotype: A double-blinded, placebo-controlled cross-over study. Br J Nutr 102: 1065-1074, 2009.

11. Larson A, Witman MA, Guo Y, Ives S, Richardson RS, Bruno RS, Jalili T and Symons JD: Acute, quercetin-induced reductions in blood pressure in hypertensive individuals are not secondary to lower plasma angiotensin-converting enzyme activity or endothelin-1: Nitric oxide. Nutr Res 32: 557-564, 2012.

12. Edwards RL, Lyon T, Litwin SE, Rabovsky A, Symons JD and Jalili T: Quercetin reduces blood pressure in hypertensive subjects. J Nutr 137: 2405-2411, 2007.

13. Pfeuffer M, Auinger A, Bley U, Kraus-Stojanowic I, Laue C Winkler P, Rüfer CE, Frank J, Bösch-Saadatmandi C, Rimbach G and Schrezenmeir J: Effect of quercetin on traits of the metabolic syndrome, endothelial function and inflammation in men with different APOE isoforms. Nutr Metab Cardiovasc Dis 23: 403-409, 2013.

14. Knab AM, Shanely RA, Henson DA, Jin F, Heinz SA, Austin MD and Nieman DC: Influence of quercetin supplementation on disease risk factors in community-dwelling adults. J Am Diet Assoc 111: 542-549, 2011

15. Kleemann R, Verschuren L, Morrison M, Zadelaar S, van Erk MJ, Wielinga PY and Kooistra T: Anti-inflammatory, anti-proliferative and anti-atherosclerotic effects of quercetin in human in vitro and in vivo models. Atherosclerosis 218: 44-52, 2011.

16. Garelnabi M, Mahini $\mathrm{H}$ and Wilson $\mathrm{T}$ : Quercetin intake with exercise modulates lipoprotein metabolism and reduces atherosclerosis plaque formation. J Int Soc Sports Nutr 11: 22, 2014.

17. Bhaskar S, Kumar KS, Krishnan K and Antony H: Quercetin alleviates hypercholesterolemic diet induced inflammation during progression and regression of atherosclerosis in rabbits. Nutrition 29: 219-229, 2013.

18. Juźwiak S, Wójcicki J, Mokrzycki K, Marchlewicz M, Białecka M, Wenda-Rózewicka L, Gawrońska-Szklarz B and Droździk M: Effect of quercetin on experimental hyperlipidemia and atherosclerosis in rabbits. Pharmacol Rep 57: 604-609, 2005 .

19. Kim JH, Kang MJ, Choi HN, Jeong SM, Lee YM and Kim JI: Quercetin attenuates fasting and postprandial hyperglycemia in animal models of diabetes mellitus. Nutr Res Pract 5: 107-111, 2011.

20. Kawai Y, Nishikawa T, Shiba Y, Saito S, Murota K, Shibata N, Kobayashi M, Kanayama M, Uchida K and Terao J: Macrophage as a target of quercetin glucuronides in human atherosclerotic arteries: Implication in the anti-atherosclerotic mechanism of dietary flavonoids. J Biol Chem 283: 9424-9434, 2008.
21. Lara-Guzman OJ, Tabares-Guevara JH, Leon-Varela YM, Álvarez RM, Roldan M, Sierra JA, Londoño-Londoño JA and Ramirez-Pineda JR: Proatherogenic macrophage activities are targeted by the flavonoid quercetin. J Pharmacol Exp Ther 343: 296-306, 2012.

22. Mao W, Fukuoka S, Iwai C, Liu J, Sharma VK, Sheu SS, Fu M and Liang CS: Cardiomyocyte apoptosis in autoimmune cardiomyopathy: Mediated via endoplasmic reticulum stress and exaggerated by norepinephrine. Am J Physiol Heart Circ Physiol 293: H1636-H645, 2007.

23. Okada K, Minamino T, Tsukamoto Y, Liao Y, Tsukamoto O, Takashima S, Hirata A, Fujita M, Nagamachi Y, Nakatani T, et al: Prolonged endoplasmic reticulum stress in hypertrophic and failing heart after aortic constriction: Possible contribution of endoplasmic reticulum stress to cardiac myocyte apoptosis. Circulation 110: 705-712, 2004.

24. Yao S, Sang H, Song G, Yang N, Liu Q, Zhang Y, Jiao P, Zong C and Qin S: Quercetin protects macrophages from oxidized low-density lipoprotein-induced apoptosis by inhibiting the endoplasmic reticulum stress-C/EBP homologous protein pathway. Exp Biol Med (Maywood) 237: 822-831, 2012.

25. Liu CM, Zheng GH, Ming QL, Sun JM and Cheng C: Protective effect of quercetin on lead-induced oxidative stress and endoplasmic reticulum stress in rat liver via the IRE1/JNK and PI3K/Akt pathway. Free Radic Res 47: 192-201, 2013.

26. Chao CL, Hou YC, Chao PD, Weng CS and Ho FM: The antioxidant effects of quercetin metabolites on the prevention of high glucose-induced apoptosis of human umbilical vein endothelial cells. Br J Nutr 101: 1165-1170, 2009.

27. Gu L, Bai W, Li S, Zhang Y, Han Y, Gu Y, Meng G, Xie L, Wang J, Xiao Y, et al: Celastrol prevents atherosclerosis via inhibiting LOX-1 and oxidative stress. PLoS One 8: e65477, 2013.

28. Urano F, Wang X, Bertolotti A, Zhang Y, Chung P, Harding HP and Ron D: Coupling of stress in the ER to activation of JNK protein kinases by transmembrane protein kinase IRE1. Science 287: 664-666, 2000.

29. Zhang K and Kaufman RJ: From endoplasmic-reticulum stress to the inflammatory response. Nature 454: 455-462, 2008.

30. Ozcan U, Yilmaz E, Ozcan L, Furuhashi M, Vaillancourt E, Smith RO, Görgün CZ and Hotamisligil GS: Chemical chaperones reduce ER stress and restore glucose homeostasis in a mouse model of type 2 diabetes. Science 313: 1137-1140, 2006.

31. Fribley A, Zhang K and Kaufman RJ: Regulation of apoptosis by the unfolded protein response. Methods Mol Biol 559: 191-204, 2009.

32. Khan MI, Pichna BA, Shi Y, Bowes AJ and Werstuck GH: Evidence supporting a role for endoplasmic reticulum stress in the development of atherosclerosis in a hyperglycaemic mouse model. Antioxid Redox Signal 11: 2289-2298, 2009.

33. Tabas I: Apoptosis and plaque destabilization in atherosclerosis: The role of macrophage apoptosis induced by cholesterol. Cell Death Differ 11 (Suppl 1): S12-S16, 2004.

34. Hansson GK: Inflammation, atherosclerosis and coronary artery disease. N Engl J Med 352: 1685-1695, 2005.

35. Han S, Liang CP, DeVries-Seimon T, Ranalletta M, Welch CL, Collins-Fletcher K, Accili D, Tabas I and Tall AR: Macrophage insulin receptor deficiency increases ER stress-induced apoptosis and necrotic core formation in advanced atherosclerotic lesions. Cell Metab 3: 257-266, 2006.

36. Duan W, Paka L and Pillarisetti S: Distinct effects of glucose and glucosamine on vascular endothelial and smooth muscle cells: Evidence for a protective role for glucosamine in atherosclerosis. Cardiovasc Diabetol 4: 16, 2005.

37. Chow JM, Shen SC, Huan SK, Lin HY and Chen YC: Quercetin, but not rutin and quercitrin, prevention of $\mathrm{H} 2 \mathrm{O} 2$-induced apoptosis via anti-oxidant activity and heme oxygenase 1 gene expression in macrophages. Biochem Pharmacol 69: 1839-1851, 2005.

38. Garige M, Gong M, Varatharajalu R and Lakshman MR: Quercetin up-regulates paraoxonase 1 gene expression via sterol regulatory element binding protein 2 that translocates from the endoplasmic reticulum to the nucleus where it specifically interacts with sterol responsive element-like sequence in paraoxonase 1 promoter in $\mathrm{HuH7}$ liver cells. Metabolism 59: 1372-1378, 2010

39. Fogelman AM, Shechter I, Seager J, Hokom M, Child JS and Edwards PA: Malondialdehyde alteration of low density lipoproteins leads to cholesteryl ester accumulation in human monocyte-macrophages. Proc Natl Acad Sci USA 77: 2214-2218, 1980. 
40. Bao L, Cai X, Zhang Z and Li Y: Grape seed procyanidin B2 ameliorates mitochondrial dysfunction and inhibits apoptosis via the AMP-activated protein kinase-silent mating type information regulation 2 homologue 1-PPAR $\gamma$ co-activator- $1 \alpha$ axis in rat mesangial cells under high-dose glucosamine. Br J Nutr 1-10, 2014 (Epub Ahead of Print).

41. Kang ES, Han D, Park J, Kwak TK, Oh MA, Lee SA, Choi S, Park ZY, Kim Y and Lee JW: O-GlcNAc modulation at Aktl Ser473 correlates with apoptosis of murine pancreatic beta cells Exp Cell Res 314: 2238-2248, 2008.

42. Ma J and Hart GW: Protein O-GlcNAcylation in diabetes and diabetic complications. Expert Rev Proteomics 10: 365-380, 2013.

43. Hawkins M, Angelov I, Liu R, Barzilai N and Rossetti L: The tissue concentration of UDP-N-acetylglucosamine modulates the stimulatory effect of insulin on skeletal muscle glucose uptake. J Biol Chem 272: 4889-4895, 1997.

44. Marshall S, Bacote V and Traxinger RR: Discovery of a metabolic pathway mediating glucose-induced desensitization of the glucose transport system. Role of hexosamine biosynthetic in the induction of insulin resistance. J Biol Chem 266: 4706-4712, 1991.

45. Burke AP, Kolodgie FD, Zieske A, Fowler DR, Weber DK, Varghese PJ, Farb A and Virmani R: Morphologic findings of coronary atherosclerotic plaques in diabetics: A postmortem study. Arterioscler Thromb Vasc Biol 24: 1266-1271, 2004.

46. Han I, Oh ES and Kudlow JE: Responsiveness of the state of $\mathrm{O}$-linked $\mathrm{N}$-acetylglucosamine modification of nuclear pore protein p62 to the extracellular glucose concentration. Biochem J 15: 109-114, 2000.

47. Roshanzamir F and Yazdanparast R: Quercetin attenuates cell apoptosis of oxidant-stressed SK-N-MC cells while suppressing up-regulation of the defensive element, HIF-1 $\alpha$. Neuroscience 277: 780-793, 2014.

48. Suganya N, Bhakkiyalakshmi E, Suriyanarayanan S, Paulm urugan R and Ramkumar KM: Quercetin ameliorates tunicamycin-induced endoplasmic reticulum stress in endothelial cells. Cell Prolif 47: 231-240, 2014.

49. Dai X, Ding Y, Zhang Z, Cai X and Li Y: Quercetin and quercitrin protect against cytokine-induced injuries in RINm5F $\beta$-cells via the mitochondrial pathway and NF- $\mathrm{KB}$ signaling. Int J Mol Med 31: 265-271, 2013

50. Xu C, Bailly-Maitre B and Reed JC: Endoplasmic reticulum stress: Cell life and death decisions. J Clin Invest 115: 2656-2664, 2005.

51. Chistiakov DA, Sobenin IA, Orekhov AN and Bobryshev YV: Role of endoplasmic reticulum stress in atherosclerosis and diabetic macrovascular complications. Biomed Res Int 610140, 2014.

52. Beriault DR, Sharma S, Shi Y, Khan MI and Werstuck GH: Glucosamine-supplementation promotes endoplasmic reticulum stress, hepatic steatosis and accelerated atherogenesis in apoE-/mice. Atherosclerosis 219: 134-40, 2011.
53. Liu CM, Zheng GH, Ming QL, Sun JM and Cheng C: Protective effect of quercetin on lead-induced oxidative stress and endoplasmic reticulum stress in rat liver via the IRE1/JNK and PI3K/Akt pathway. Free Radic Res 47: 192-201, 2013.

54. Xu LH, Xie H, Shi ZH, Du LD, Wing YK, Li AM, Ke Y and Yung WH: Critical role of endoplasmic reticulum stress in chronic intermittent hypoxia-induced deficits in synaptic plasticity and long-term memory. Antioxid Redox Signal, May 8 , 2015 (Epub ahead of print).

55. van der Kallen CJ, van Greevenbroek MM, Stehouwer CD and Schalkwijk CG: Endoplasmic reticulum stress-induced apoptosis in the development of diabetes: Is there a role for adipose tissue and liver? Apoptosis 14: 1424-1434, 2009.

56. Minamino T and Kitakaze M: ER stress in cardiovascular disease. J Mol Cell Cardiol 48: 1105-1110, 2010.

57. Zinszner H, Kuroda M, Wang X, Batchvarova N, Lightfoot RT, Remotti H, Stevens JL and Ron D: CHOP is implicated in programmed cell death in response to impaired function of the endoplasmic reticulum. Genes Dev 12: 982-995, 1998.

58. Tsukano H, Gotoh T, Endo M, Miyata K, Tazume H, Kadomatsu T, Yano M, Iwawaki T, Kohno K, Araki K, et al: The endoplasmic reticulum stress-C/EBP homologous protein pathway-mediated apoptosis in macrophages contributes to the instability of atherosclerotic plaques. Arterioscler Thromb Vasc Biol 30: 1925-1932, 2010.

59. Hu P, Han Z, Couvillon AD, Kaufman RJ and Exton JH: Autocrine tumor necrosis factor alpha links endoplasmic reticulum stress to the membrane death receptor pathway through IRE1alpha-mediated NF-kappaB activation and down-regulation of TRAF2 expression. Mol Cell Biol 26: 3071-3084, 2006.

60. Chen S, Zhao Y, Zhang Y and Zhang D: Fucoidan induces cancer cell apoptosis by modulating the endoplasmic reticulum stress cascades. PLoS One 9: e108157, 2014

61. Nakagawa T, Zhu H, Morishima N, Li E, Xu J, Yankner BA and Yuan J: Caspase-12 mediates endoplasmic-reticulum-specific apoptosis and cytotoxicity by amyloid-beta. Nature 403: 98-103, 2000.

62. Tabas I: The role of endoplasmic reticulum stress in the progression of atherosclerosis. Circ Res 107: 839-850, 2010.

63. Yamamoto K, Sato T, Matsui T, Sato M, Okada T, Yoshida H, Harada A and Mori K: Transcriptional induction of mammalian ER quality control proteins is mediated by single or combined action of ATF6alpha and XBP1. Dev Cell 13: 365-376, 2007.

64. Yamamoto K, Takahara K, Oyadomari S, Okada T, Sato T, Harada A and Mori K: Induction of liver steatosis and lipid droplet formation in ATF6alpha-knockout mice burdened with pharmacological endoplasmic reticulum stress. Mol Biol Cell 21: 2975-2986, 2010

65. Rutkowski DT, Wu J, Back SH, Callaghan MU, Ferris SP, Iqbal J, Clark R, Miao H, Hassler JR, Fornek J, et al: UPR pathways combine to prevent hepatic steatosis caused by ER stress-mediated suppression of transcriptional master regulators. Dev Cell 15: 829-840, 2008. 